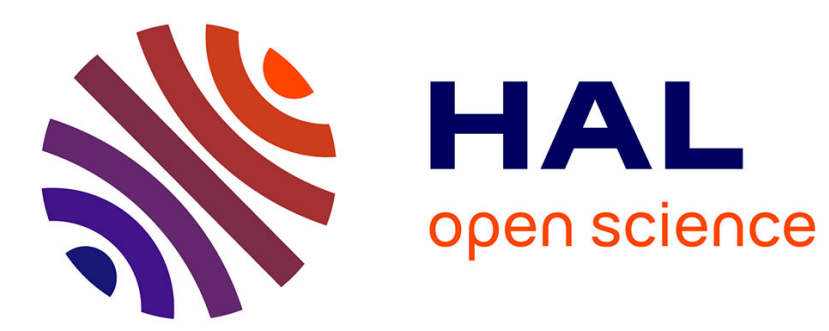

\title{
An Adaptively Reduced Order Extended Kalman Filter for Data Assimilation in the Tropical Pacific
}

\author{
Ibrahim Hoteit, Dinh-Tuan Pham
}

\section{To cite this version:}

Ibrahim Hoteit, Dinh-Tuan Pham. An Adaptively Reduced Order Extended Kalman Filter for Data Assimilation in the Tropical Pacific. [Research Report] RR-4298, INRIA. 2001. inria-00072289

\section{HAL Id: inria-00072289 \\ https://hal.inria.fr/inria-00072289}

Submitted on 23 May 2006

HAL is a multi-disciplinary open access archive for the deposit and dissemination of scientific research documents, whether they are published or not. The documents may come from teaching and research institutions in France or abroad, or from public or private research centers.
L'archive ouverte pluridisciplinaire HAL, est destinée au dépôt et à la diffusion de documents scientifiques de niveau recherche, publiés ou non, émanant des établissements d'enseignement et de recherche français ou étrangers, des laboratoires publics ou privés. 


\section{N R I A}

INSTITUT NATIONAL DE RECHERCHE EN INFORMATIQUE ET EN AUTOMATIQUE

\section{An adaptively reduced order extended Kalman filter for data assimilation in the tropical Pacific}

Ibrahim Hoteit and Dinh-Tuan Pham

No 4298

Octobre 2001

THÈME 4 



\title{
RIN RIA
}

\section{An adaptively reduced order extended Kalman filter for data assimilation in the tropical Pacific}

\author{
Ibrahim Hoteit and Dinh-Tuan Pham \\ Thème 4 - Simulation et optimisation \\ de systèmes complexes \\ Projet IDOPT
}

Rapport de recherche $\mathrm{n}^{\circ} 4298$ - Octobre 2001 - 25 pages

\begin{abstract}
The reduced-order extended Kalman (ROEK) filter has been introduced by Cane et al. [3] as a means to reduce the cost of the extended Kalman (EK) filter. It essentially consists in projecting the dynamic of the model onto a low dimensional subspace obtained via an empirical orthogonal functions (EOF) analysis. However, the choice of the dimension of the reduced state space (or the number of EOFs to be retained) remains a delicate question. Indeed, Cane et al. [3] have been surprised by the fact that increasing the number of EOFs does not improve, and even sometimes worsen, the performance of the ROEK filter. We suspect that it is due to the optimal character of the EOF analysis which is optimal in a timemean sense only. In this respect, we develop a simple efficient adaptive scheme to tune, according to the model mode, the dimension of the reduced state space, which would be variable in time. In a first application, twin experiments are conducted in a realistic setting of the OPA model in the tropical pacific. The observations are assumed to be synthetic altimeter data sampled according to the Topex/Poseidon mission features. Our adaptive scheme is shown to improve the performance of the ROEK filter especially during the model unstable periods.
\end{abstract}

Key-words: Data assimilation. OPA model. Kalman filter. ROEK Filter. EOF analysis. Forgetting factor.

(Résumé : tsvp)

This work was carried out within the framework of the IDOPT project which is a joint project between INRIA, CNRS, University Joseph Fourier and INPG. 


\section{Un filtre de Kalman d'ordre réduit adaptatif pour l'assimilation de données dans le tropical pacifique}

Résumé : Le filtre de Kalman étendu d'ordre réduit (ROEK) a été introduit par Cane et al. [3] comme une solution pour réduire le coût du filtre de Kalman étendu (EK). Il consiste essentiellement à projeter la dynamique du modèle sur un sousespace de dimension faible obtenu à partir d'une analyse empirique orthogonale (EOF). Cependant, le choix de la dimension de l'espace d'état réduit (ou le nombre de EOFs à retenir) reste toujours une question delicate. En effet, Cane et al. [3] ont été surpris de remarquer que le fait d'augmenter le nombre de EOFs n'améliore pas, voir même détériore parfois, la performance du filtre ROEK. Nous soupçonnons qu'elle est due au critère d'optimalité de l'analyse EOF qui est optimale uniquement au sens d'une moyenne temporelle. A cet égard, nous développons un schéma adaptatif simple et efficace pour régler, selon le régime du modèle, la dimension de l'espace d'état réduit, qui peut être variable dans le temps. En première application, des expériences jumelles ont été conduites dans une configuration réalise du modèle OPA dans l'océan tropical pacifique. Les observations ont été supposées altimétriques selon les traces du satellite Topex/Poseidon. Notre shéma adaptatif s'est montré capable d'améliorer la perfromance du filtre ROEK, particulièrement durant les périodes instables du modèle.

Mots-clé : Assimilation de données. Modèle OPA. Filtre de Kalman. Filtre ROEK. Analyse EOF. Facteur d'oubli. 


\section{Introduction}

The goal of data assimilation is to construct estimate of the state of a dynamical system by combining both the information from a numerical model and from the observations. This problem has been first study in the context of meteorology but have recently attracted much attention in oceanography, thanks to several satellite observation missions which provided a large number of measurements, and also to the continuous progress in computing power. The assimilation methods can be classified in two principal categories: sequential methods based on the statistical estimation theory and variational methods based on the optimal control theory (see Ghil and Manalotte-Rizzoli [10] for a review). The present work concerns the domain of statistical methods, in particular the well known Kalman filter.

The Kalman filter provides the best linear unbiased estimate, in the sense of least-squares, of the ocean state using all observations available up to the analysis time [15]. It is easy to implement but its application into realistic ocean models encounters two major difficulties: non-linearity and computational cost. The first can be partially resolved by linearizing the model around the state estimate, which leads to the so called extended Kalman (EK) filter [14]. The second is due to the huge dimension of the model state. Several variants of the EK filter, which essentially consist in projecting the system state via an order-reduction operator onto a low dimensional sub-space, have been proposed to reduce the dimension of the system $[5,7,8,9,18]$. A promising approach has been proposed by Cane et al. [3] who reduced the state space to a small set of basis function, using an empirical orthogonal function (EOF) analysis. We shall refer to this filter as the reduced-order extended Kalman (ROEK) filter.

In the ROEK filter, the dimension of the reduced state space (or the number of EOFs to be retained) was chosen according to the variability explained by the first EOFs (or inertia) and also to keep the cost of the filter reasonable. Cane et al. [3] expected that the higher the number of retained EOF the more performance the filter would be, since the reduced state space generated by the EOFs better represents, in some sense, the variability of the model. However, their numerical experiments reveals a surprising feature: increasing the number of EOFs does not improve, and even sometimes worsen, the performance of the filter.

The same phenomena has also been observed in our numerical experiments. A plausible explanation is that the optimality characteristic of the EOF analysis is

$\operatorname{RR} n^{\circ} 4298$ 
true in a time-mean sense only as such an analysis is based on a long historical run. The last observation motivate us to develop a simple adaptive scheme to tune the dimension of the reduced state space. Our idea consists simply in fixing a number of EOFs sufficient to represent the variability of the system in the stable period and then add some new EOFs when model instabilities appear in order to represent more completely the local structures of the model. Note that a similar approach has been already successfully implemented by Hoteit et al. [12] to tune the forgetting factor and the evolution of the correction basis, for the singular evolutive extended Kalman (SEEK) filter and its variants.

The outline of this paper is as follows. In the next section we briefly review the ROEK filter. Section 3 recalls the EOF analysis and discuss its characteristics. Section 4 describes our adaptive approach to tune the dimension of the reduced state space. Finally, section 5 explains our implementation strategy of the adaptive ROEK filter to assimilate altimetric data in the OPA model and presents the simulation results of some twin experiments conducted in the tropical pacific ocean.

\section{The ROEK filter}

We shall adopt the notation proposed by Ide et al. [13]. Consider a physical system described by

$$
X^{t}\left(t_{k}\right)=M\left(t_{k-1}, t_{k}\right) X^{t}\left(t_{k-1}\right)+\eta\left(t_{k}\right),
$$

where $X^{t}(t)$ is a vector representing the true state at time $t, M(s, t)$ is an operator describing the system transition from time $s$ to time $t$ and $\eta(t)$ is the system noise vector. At each time $t_{k}$, the state vector is observed by the system

$$
Y_{k}^{o}=H_{k} X^{t}\left(t_{k}\right)+\varepsilon_{k},
$$

where $H_{k}$ is the observational operator and $\varepsilon_{k}$ is the observational noise. The noises $\eta\left(t_{k}\right)$ and $\varepsilon_{k}$ are assumed to be independent random vectors with mean zero and covariance matrices $Q_{k}$ and $R_{k}$, respectively.

The sequential data assimilation consists in the estimation of the state system $X^{t}$ at each observation time, using observations up to this time. In the linear case, this problem has been entirely solved by the well known Kalman filter. This filter possesses an attractive feature of being recursive. Computation is done "on line" as 
soon as new observations are available. In the nonlinear case, one often linearizes the model around the current estimated state vector, which yields to the so called extended Kalman EK filter (see for example Ghil and Manalotte-Rizzoli [10] for a review). Apart from initialization, this filter proceeds as a succession of forecasting and correction steps. Assuming that at a time $t_{k-1}$, one already has an estimate of the system state, often referred to as the analysis state vector $X^{a}\left(t_{k-1}\right)$, with some analysis error covariance matrix $P^{a}\left(t_{k-1}\right)$. The EK filter allows the construction of the next $X^{a}\left(t_{k}\right)$ by correcting the forecast $X^{f}\left(t_{k}\right)$, which is the output of the model starting from $X^{a}\left(t_{k-1}\right)$, using the new observation. It also provides the calculation of the new analysis error covariance matrix $P^{a}\left(t_{k}\right)$, to reflect both the propagation of error from the analysis to the forecasting step, resulting in an error covariance matrix $P^{f}\left(t_{k}\right)$, and the reduction of error achieved by the correction step. The reader can consult Jazwinski [14] for more details.

In the oceanic models, the dimension of the state vector is of the order $10^{7}$. The use of the EK filter to assimilate data in these models can not thus be done without massive order reduction, since otherwise the size of $P^{a}$ would be about $10^{7} \times 10^{7}$. As proposed by Fukumori et al. [9], the use of a (linear) reduction operator, which relates the state vector of the system to a small dimension reduced-order state vector, provides us several alternatives to reduce the cost of the EK filter. Indeed, this enables us to avoid the evolution of $P^{a}$ and $P^{f}$ by letting the forecast error to evolve in the reduced-order state space, after it has been "transported" in this space, and then to reconstruct this error in its origin (or full) space via the pseudo-inverse of the reduction operator. One obtains in this manner the equations of the reduced order extended Kalman (ROEK) filter which is the most used variant of the EK filter in practice (De Mey [6]).

Without lost of generality, one can assume that the reduction operator $S$ is orthogonal so that its pseudo-inverse is equal to $S^{T}$. The full state vector $X^{t}$ is then related to the reduced state vector $X_{r}^{t}$ by

$$
X^{t}=S X_{r}^{t}
$$

If this assumption is used in the EK filter, one obtains the equations of the ROEK filter which operates in two stages apart from an initialization stage as the EK filter (see [9] for more details).

$\operatorname{RR} n^{\circ} 4298$ 
0- Initialization stage: We resort here to an objective analysis, based on the first observation $Y_{0}^{O}$ : we take as the initial analysis state vector

$$
X^{a}\left(t_{0}\right)=\bar{X}+S P_{r}^{a}\left(t_{0}\right) S^{T} \mathbf{H}_{0}^{T} R_{0}^{-1}\left[Y_{0}^{o}-H_{0} \bar{X}\right]
$$

where

$$
P_{r}^{a}\left(t_{0}\right)=\left[S^{T} \mathbf{H}_{0}^{T} R_{0}^{-1} \mathbf{H}_{0} S\right]^{-1},
$$

$\bar{X}$ is the average of a sequence of state vectors and $\mathbf{H}_{0}$ is the gradient of $H_{0}$ evaluated at $\bar{X}$. The initial analysis error covariance matrix may be taken as

$$
P^{a}\left(t_{0}\right)=S P_{r}^{a}\left(t_{0}\right) S^{T} .
$$

Note that we have used the first observation for initialization, the algorithm actually starts with the next observation.

1- Forecast stage: One applies the model (1) to compute the forecast state

$$
X^{f}\left(t_{k}\right)=M\left(t_{k}, t_{k-1}\right) X^{a}\left(t_{k-1}\right)
$$

and let the covariance matrix of the forecast error in the reduced space $P_{r}^{f}\left(t_{k}\right)$ (of dimension $r \times r$ ) to evolve according to

$$
P_{r}^{f}\left(t_{k}\right)=\left[S^{T} \mathbf{M}\left(t_{k}, t_{k-1}\right) S\right] P_{r}^{a}\left(t_{k-1}\right)\left[S^{T} \mathbf{M}\left(t_{k}, t_{k-1}\right) S\right]^{T}+S^{T} Q_{k} S .
$$

The forecast error covariance matrix is then equal to

$$
P^{f}\left(t_{k}\right)=S P_{r}^{f}\left(t_{k}\right) S^{T} .
$$

2- Correction stage: The correction of the forecast state is done according to the formula

$$
X^{a}\left(t_{k}\right)=X^{f}\left(t_{k}\right)+G_{k}\left[Y_{k}^{o}-H_{k} X^{f}\left(t_{k}\right)\right]
$$

where $G_{k}$ is given by

$$
G_{k}=S P_{r}^{f}\left(t_{k}\right) S^{T} \mathbf{H}_{k}^{T} R_{k}^{-1} .
$$

The covariance matrix of the analysis error covariance matrix $P_{r}^{a}\left(t_{k}\right)$ is then updated from the equation

$$
\left[P_{r}^{a}\left(t_{k}\right)\right]^{-1}=\left[P_{r}^{f}\left(t_{k}\right)\right]^{-1}+S^{T} \mathbf{H}_{k}^{T} R_{k}^{-1} \mathbf{H}_{k} S .
$$

The analysis error covariance matrix is then given by

$$
P^{a}\left(t_{k}\right)=S P_{r}^{a}\left(t_{k}\right) S^{T} .
$$


It is important to notice here that we have neglected in formula (3) the representativeness error which should represent the information that has not been explained by the reduced space defined by $S$ (this error can be conveniently "inserted" into the model error (see [3])). However, following Pham et al. [19], we introduce instead the use of a forgetting factor to limit the propagation of this error with time. This approach has been adopted as a way to sidestep the difficulty of correctly specifying the representativeness error. The equations of the ROEK filter algorithm with the forgetting factor remains unchanged. Only the update equation of the analysis error covariance matrix in the reduced space is replaced by

$$
\left[P_{r}^{a}\left(t_{k}\right)\right]^{-1}=\rho\left[P_{r}^{f}\left(t_{k}\right)\right]^{-1}+S^{T} \mathbf{H}_{k}^{T} R_{k}^{-1} \mathbf{H}_{k} S .
$$

Concerning the cost of this filter, it mostly comes from the calculation of the evolution equation (7) of the forecast error in the reduced state space. It thus depends on the dimension of the reduced space $r$ because the numerical calculation of $\mathbf{M}\left(t_{k}, t_{k-1}\right) S$ requires $(r+1)$ integrations of the tangent linear model. A reasonably low choice of $r$ is then imperative for realistic applications.

The performance of the ROEK filter highly depends on the representativeness of the reduction operator $S$. A good choice of $S$ should lead to a large reduction of the dimension of the system and to a reduced state space which well represents the variability of the model. Different forms of the operator $S$ have been proposed in the literature such as the use of a coarse resolution [8], the most dominant singular modes of the tangent linear model and the most dominant eigenmodes of the analysis error covariance matrix [4], etc, which are supported by more or less simplifying assumptions on the dynamic and the characteristics of the model (see De Mey [6] for a review).

With the same aim in view, Cane et al. [3] have adopted a different approach: using the empirical orthogonal functions (EOF) analysis, they reduced the state space for the forecast covariance updated to a small set of basis functions, called EOFs, which nonetheless represented all the significant structures that were predicted by the model. More than the implementation cost reasons, the philosophy of order reduction of Cane et al. [3] relies on the fact that since we can not precisely compute the "true" error covariance matrix, it is useless to try to specify its full description. In numerical applications, this procedure was shown to lead to a substantial saving without any loss of accuracy compared to the full EK filter [3]. 


\section{$3 \quad$ EOF analysis}

This analysis aims at providing a representation as accurately as possible of a sample of state vectors $X_{1}, \ldots, X_{N}$ in $\mathbb{R}^{n}$ in a low-dimension (denoted $r$ ) subspace. For a vector $X$, let $\tilde{X}$ denotes its orthogonal projection onto a subspace of dimension $r$ spanned by an $\mathcal{M}$-orthogonal basis $S=\left\{\phi_{k}\right\}_{k=1, \ldots, r}, \mathcal{M}$ being some metric (to be chosen) in the state space, and the constant function

$$
\tilde{X}=\bar{X}+\sum_{k=1}^{r} c_{k} \phi_{k}=\bar{X}+S S^{T} \mathcal{M}(X-\bar{X})
$$

where $\bar{X}=\frac{1}{N} \sum_{i=1}^{N} X_{i}$ is the average of $X_{1}, \ldots, X_{N}$ and

$$
c_{k}=\left\langle X-\bar{X}, \phi_{k}\right\rangle_{\mathcal{M}}=\phi_{k}^{T} \mathcal{M}(X-\bar{X}) .
$$

The EOF analysis then consists of minimizing the mean squares projection error

$$
e^{2}=\frac{1}{N} \sum_{i=1}^{N}\left\|X_{i}-\tilde{X}_{i}\right\|_{\mathcal{M}}^{2}
$$

with respect to all choices of the basis. Here the introduction of a metric $\mathcal{M}$ is needed in the case where the state variables are not homogeneous (as they represent different physical variable such as velocity, salinity, temperature ...) to define a distance between state vectors independent from unit of measure.

The solution to the above minimization problem is given by the first $r$ normalized eigenvectors $\phi_{1}, \ldots, \phi_{r}$ of the sample covariance matrix $P$ of $X_{1}, \ldots, X_{N}$, namely

$$
P=\frac{1}{N} \Omega \Omega^{T} \quad \text { with } \quad \Omega=\left[X_{1}-\bar{X} \cdots X_{N}-\bar{X}\right],
$$

relative to the metric $\mathcal{M}$, the eigenvectors being ranked in decreasing order of their eigenvalues $\lambda_{1}, \ldots, \lambda_{r}$. With regard to the choice of $r$, it has been shown that the fraction of variance (or inertia) explained by the first $r$ EOFs is given by

$$
\mathcal{I}\left(\phi_{1}, \ldots, \phi_{r}\right)=\frac{\sum_{k=1}^{r} \lambda_{k}}{\sum_{k=1}^{p} \lambda_{k}},
$$

and thus can be used as a guide for choosing $r$ (this fraction should be close to 1). The reader is referred to Preisendorfer [21] for more details. 
In our case, we are interested in representing the variability of the state model around its mean and thus we use a long historical sequence of model states $X_{1}, \ldots, X_{N}$ which can be extracted from a model run. Therefore, the matrix $P$ contains a bulk of information on the system variability when $N$ is sufficiently large.

\section{Adaptive tuning of the dimension of the reduced space}

As explained in the above section, the dimension $r$ of the reduced state space (or the number of EOFs to be retained) was chosen according to the value of $\mathcal{I}$, providing that the cost of this filter remains reasonable since this cost highly depends on this number. Cane et al. [3] have noticed in their numerical experiments that increasing the number of EOFs does not improve, and even sometimes worsen, the performance of the filter. The same phenomena has been also observed in our numerical experiments. For a plausible explanation, we observe that the optimality property of the EOF analysis is only optimal (at best) in a time-mean sense. Indeed, the EOF analysis is done over a long time period composed of periods in which the system evolves stably and periods in which it evolves unstably. Local perturbations often arise in the latter period and not in the former, and they are represented by the last EOFs corresponding to the last eigenvalues [3, 11]. Using more EOFs when the system is in a stable period would introduce spurious information which can degrade the performance of the filter. On the other hand, using only a few EOFs would not be enough to represent all the local structures of the system in the unstable periods. The filter will then discard corrections in these structures and the error may grow. Therefore to achieve better performance of the ROEK filter, our idea is quite simply and consists in fixing a number of EOFs suitable to the stable period and then add some EOFs in the unstable periods in order to represent more completely the model local structures. In other words, we will give the dimension $r$ of the reduced state space one of two values $r_{1}$ and $r_{2}\left(r_{1}<r_{2}\right)$ according to the model state.

Such an adaptive scheme can be easily implemented in the ROEK filter. Indeed, if one denotes by $S_{1}$ and $S_{2}$ the basis containing the first $r_{1}$ and $r_{2}$ EOFs respectively, the algorithm of the ROEK filter is taken to be the same as described in section 2, using $S_{1}$ (instead of $S$ ) when the model is stable and $S_{2}$ when model instabilities appear. However, in the transition phase "stable to unstable" and vice-versa, one has to change the equation (7). Specifically, it is replaced by

$$
P_{r}^{f}\left(t_{k}\right)=\left[S_{2}^{T} \mathbf{M}\left(t_{k}, t_{k-1}\right) S_{1}\right] P_{r}^{a}\left(t_{k-1}\right)\left[S_{2}^{T} \mathbf{M}\left(t_{k}, t_{k-1}\right) S_{1}\right]^{T}+S_{2} Q S_{2}^{T}
$$

$\operatorname{RR} n^{\circ} 4298$ 
each time the model goes from a stable to an unstable period (i.e. $S_{2}$ is used instead of $S_{1}$ ), meaning that the reduced forecast error $e_{r}^{f}\left(t_{k}\right)=M\left(t_{k}, t_{k-1}\right) S_{1} e_{r}^{a}\left(t_{k-1}\right)$ (where $e_{r}^{a}\left(t_{k}\right)$ is the reduced analysis error) is projected onto the subspace generated by $S_{2}$, and by

$$
P_{r}^{f}\left(t_{k}\right)=\left[S_{1}^{T} \mathbf{M}\left(t_{k}, t_{k-1}\right) S_{2}\right] P_{r}^{a}\left(t_{k-1}\right)\left[S_{1}^{T} \mathbf{M}\left(t_{k}, t_{k-1}\right) S_{2}\right]^{T}+S_{1} Q S_{1}^{T} .
$$

when model instabilities vanish $\left(S_{1}\right.$ is used instead of $\left.S_{2}\right)$.

A similar approach has been already adopted by Hoteit et al. [11, 12] to adapt the forgetting factor and the evolution of the correction basis for the SEEK filter and its variants. To detect the model unstable periods, Hoteit et al. $[11,12]$ proposed to track the filter's behavior by computing an instantaneous average and a long term average of the prediction error variance, denoted by $s_{k}$ and $l_{k}$ respectively. Therefore, if $c s_{k} \leq l_{k}$ ( $c$ is a tuning constant), they assumed steady conditions have been achieved and considered that the model is in a stable period. In this case we use $r=r_{1}$ EOFs. Otherwise, if $c s_{k}>l_{k}$, this is an indication that the model may be in an unstable period since this would degrade the filter short term performance (the long term performance is weakly affected because it is averaged over a long duration). We must then increase the number of EOFs and thus take $r=r_{2}$ EOFs.

Estimates of $s_{k}$ and $l_{k}$ are computed recursively as in Hoteit et al. [12]:

$$
\begin{aligned}
& s_{k}=\alpha s_{k-1}+(1-\alpha)\left\|Y_{k}^{o}-H_{k} X^{f}\left(t_{k}\right)\right\|^{2}, \\
& l_{k}=\beta l_{k-1}+(1-\beta)\left\|Y_{k}^{o}-H_{k} X^{f}\left(t_{k}\right)\right\|^{2}
\end{aligned}
$$

where $\alpha$ and $\beta$ are constants chosen such as $\beta \lesssim 1$ and $\alpha<\beta$.

Note that Hoteit et al. [12] have used the same approach to tune the value of the forgetting factor by giving it one of two values $\rho_{1} \lesssim 1$ and $\rho_{2}<\rho_{1}$. One can therefore use this adaptive scheme together with the one on the number of retained EOFs.

\section{Application to altimetric data assimilation in the OPA model of the tropical Pacific}

To evaluate the performance of our adaptive tuning scheme described in the above section, we have implemented the ROEK filter in a realistic setting of the OPA model 
in the tropical Pacific ocean, under the assumption of a perfect model $\left(Q_{k}=0\right)$. The assimilation is based on the pseudo-observations which are extracted from twin experiments.

\subsection{Model description}

\subsubsection{The OPA model}

The OPA model (OPA for Océan PArallélisé) is a primitive equation ocean general circulation model which has been developed at the LODYC laboratory (Laboratoire d'Océanographie DYnamique et de Climatologie) to study large scale ocean circulation. It solves the Navier-Stokes equations plus the rigid lid assumption and some hypothesis made from scale considerations. The system equations is written in curvilinear z-coordinates and discretized using the centered second order finite difference approximation on a three dimension generalized "C-grid Arakawa" (see Arakawa [1] for details). Time stepping is achieved by two time differencing schemes: a basic leap-frog scheme associated to an Asselin filter for the non-diffusive processes and a forward scheme for diffusive terms. The sub-grid scale physics are a tracer diffusive operators of second order on the vertical, the eddy coefficients being computed from a turbulent closure model (see Blanke and Delecluse [2]). On the lateral, diffusive and viscous operators can be either of second or of fourth order. The reader is referred to the OPA reference manual Madec et al. [17] for more details.

\subsubsection{Configuration of the tropical Pacific}

The model domain covers the entire tropical Pacific basin extending from $120^{\circ} \mathrm{E}$ to $70^{\circ} \mathrm{W}$ and from $33^{\circ} \mathrm{S}$ to $33^{\circ} \mathrm{N}$ and the level depth varies from 0 at the sea surface to $4000 \mathrm{~m}$. Two buffer zones are included between $20^{\circ}$ and $33^{\circ}$ in the north and south of the domain, for the connection with the sub-tropical gyres. The number of horizontal grid points is $171 \times 59$ on 25 vertical levels. The model equations are solved on an horizontal grid with a zonal resolution $1^{\circ}$ and a meridional resolution maximum at the equator of $0.5^{\circ}$ and goes down to $2^{\circ}$ to north and south boundaries. The vertical resolution is approximatively $10 \mathrm{~m}$ from the sea surface to $120 \mathrm{~m}$ depth then decreases to $1000 \mathrm{~m}$ at the sea bottom. The time step is one hour.

The bathymetry is relatively coarse. It was obtained from Levitus data's mask [16]. The forcing fields are interpolated from the ECMWF reanalysis with monthly variability. It is composed of wind stress and heat, temperature and fresh water fluxes. Zero fluxes of heat and salt and non-slip conditions are applied at solid 
boundaries. A second order horizontal friction and diffusion scheme for momentum and tracers is chosen with a coefficient of $2000 \mathrm{~m}^{2} / \mathrm{s}$ in the strip $10^{\circ} \mathrm{N}-10^{\circ} \mathrm{S}$ and increase up to $10000 \mathrm{~m}^{2} / \mathrm{s}$ at the north and south basins boundaries. The static instabilities are resolved in the turbulent closure scheme. The model starts from rest (i.e. with zero velocity field). The salinity and the temperature are stem from seasonal climatologic Levitus data [16].

\subsubsection{The state vector}

The state vector $X^{t}$ is the set of prognostic variables, which, in the OPA model, consists of the zonal and meridional velocity $U$ and $V$, the salinity $S$ and temperature $T$, thus

$$
X^{t}=(U, V, S, T)^{T} .
$$

However, the observation, which is the sea surface height $(S S H)$, is directly related to the surface pressure $P_{s}$ but the later only indirectly related to the state variables through a set of partial differential equations. More precisely, $P_{s}$ is a diagnostic variable which can be computed through the system of equations (Pinardi et al. [20])

$$
\nabla\left(H \nabla P_{s}\right)=-\nabla(f H \mathbf{k} \wedge \mathbf{u})-\nabla \int_{-H}^{0}(z+H) \nabla \rho d z+\varepsilon \nabla \mathbf{B}
$$

where $\nabla$ denotes the horizontal gradient, $H$ is the ocean depth, $f$ is the Coriolis factor, $\mathbf{k}$ is the vertical unit vector, $\mathbf{u}$ is the horizontal vector velocity field, $\rho$ is the density, $\varepsilon$ is the Rossby number and $\mathbf{B}$ is a term describing the nonlinear advection and dissipation effects. The term $\mathbf{B}$ is rather complex but is also needed for solving the system equations concerning $X^{t}$. In fact the numerical code for integrating the OPA model also computes, as a by product, the diagnostic variable $P_{s}$. Therefore, for purely technical reasons, it is advantageous to augment the state vector by including the variable $P_{s}$, that is we now take

$$
X^{t}=\left(U, V, S, T, P_{s}\right)^{T} .
$$

Of course, the model equations must now include the extra equation (24) and the derivation of our filter algorithm must be based on this extended model, not on the original model (the EOF analysis are also carried out on the augmented state vectors). The overhead is insignificant anyway since $P_{s}$ is only defined on the surface of the ocean. The number of state variables increases from $4 \times 171 \times 59 \times 25=1008900$ 
to $4 \times 171 \times 59 \times 25+171 \times 59=1018989$. The numerical cost of the algorithm would increase by the same proportion since this cost is roughly proportional to the dimension of the state vector. This increase would be offset by the fact that the calculation of the observation operator is now straightforward. If we work with the original state vector, we would have to pull out the portions of the OPA code for computing directly $P_{s}$ from $(U, V, S, T)^{T}$, with some unavoidable redundancy with the integration of the model equations. But the main point is that this would increase considerably the complexity of the programming work without incurring real differences, both in term of cost and in term of methodology.

\subsection{Data and filters validation}

Twin experiments are used to assess the performances and the capabilities of our adaptive scheme. Therefore, a reference experiment is performed and the reference state $X^{t}$ retained to be later compared with the fields produced by the filter. More precisely, a sequence of 250 state vectors was retained every 3 days (which corresponds to the Topex/Poseidon ( $\mathrm{T} / \mathrm{P}$ ) measurements frequency) during the period of march $1^{\text {st }} 1991$ to november $10^{\text {st }} 1991$.

The assimilation experiments are performed using the pseudo-measurements which are extracted from the reference states. The $S S H$ is assumed to be observed according to the real tracks of $\mathrm{T} / \mathrm{P}$ with a nominal accuracy of $3 \mathrm{~cm}$. The orbital period of this satellite is 9.8 days with an observation every three days. For our numerical experiments, we produced 3 masks (see Figure 1) with a temporal interval of 3 days which we used successively. Therefore, the period of simulated observations is $3 * 3=9$ days which is different form the true period (9.8 days). However, this doesn't matter since twin experiments are performed. The observation error is simulated by adding randomly generated Gaussian noise to the synthetic observations of $S S H$. Note that in the assimilation interval, a period of very strong model instability occurs between July and September (see Figure 2)

The performance of all our filters is evaluated by comparing the relative root mean square $(R R M S)$ error for each state variable, in each layer or in the whole domain of the ocean model. The RRMS is defined as

$$
\operatorname{RRMS}\left(t_{k}\right)=\frac{\left\|X^{t}\left(t_{k}\right)-X^{a}\left(t_{k}\right)\right\|}{\left\|X^{t}\left(t_{k}\right)-\bar{X}\right\|}
$$

$\mathrm{RR} \mathrm{n}^{\circ} 4298$ 

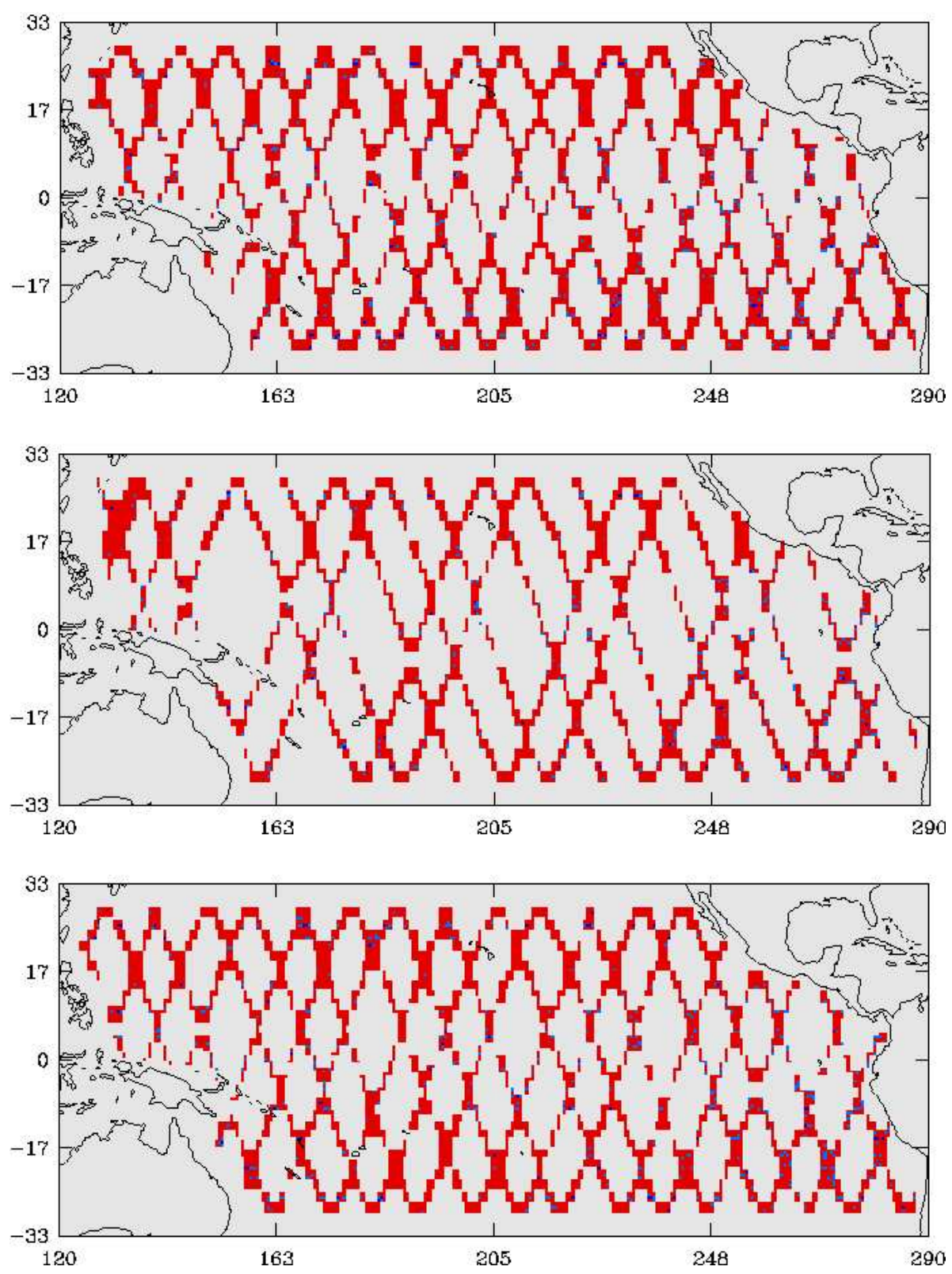

Figure 1: Topex/Poseidon satellite tracks with an orbital period of 9 days and an observation every 3 days. 


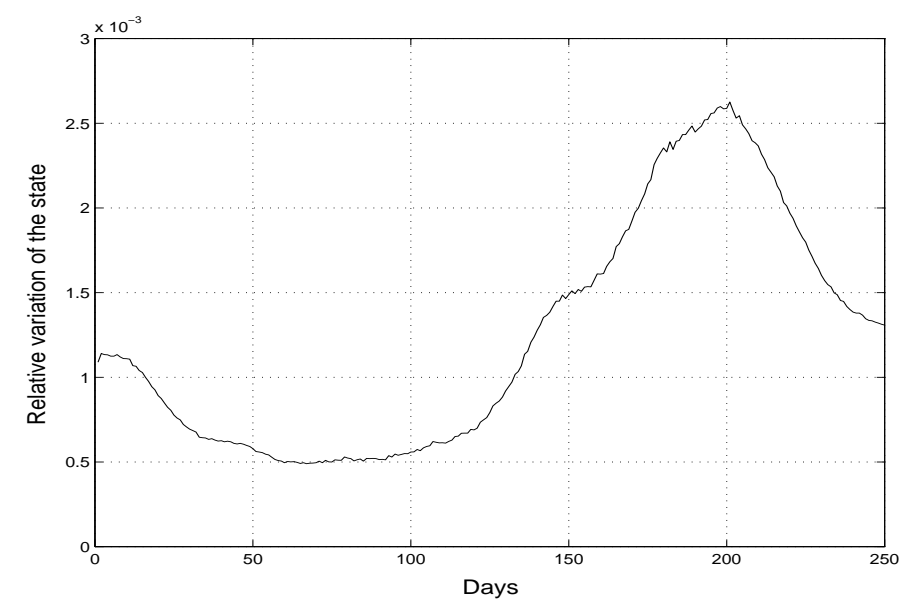

Figure 2: Relative variation of the state vector in the assimilation period.

where $\bar{X}$ is the mean state of the sample $H_{S}$ and $\|\cdot\|$ denotes the Euclidien norm. Thus the error is relative to the free-run error since the denominator represents the error when there is no observation and the analysis vector is simply taken as the mean state vector.

\subsection{Design and result of the EOF analysis}

For the present study, the data for the assimilation experiments is again simulated but in an unrelated way with the above simulation. In a first experiment, the model has been spun up for 7 years from 1980 to 1986 with the aim to reach a statistically steady state of mesoscale turbulence. Next, another integration of 4 years is carried out from 1987 to 1990 to generate a historical sequence $H_{S}$ of model realization. A sequence of 480 state vectors was retained by storing 1 state vector every 3 days to reduce the calculation since successive states are quite similar. Because the state variables in (25) are not of the same nature, we shall in fact apply a multivariate EOF analysis. Each state variable, namely $U, V, S, T$ and $P_{S}$, will be normalized by dividing it with the spatial average (over the grid points) of its variance.

Figure 3 plots the number of (global) EOFs and the percentage of inertia contained in the sample $H_{S}$ they explain. From this result, we have chosen to retain $r_{1}=35$ global EOFs in our assimilation experiments, as this achieve $87 \%$ of the 


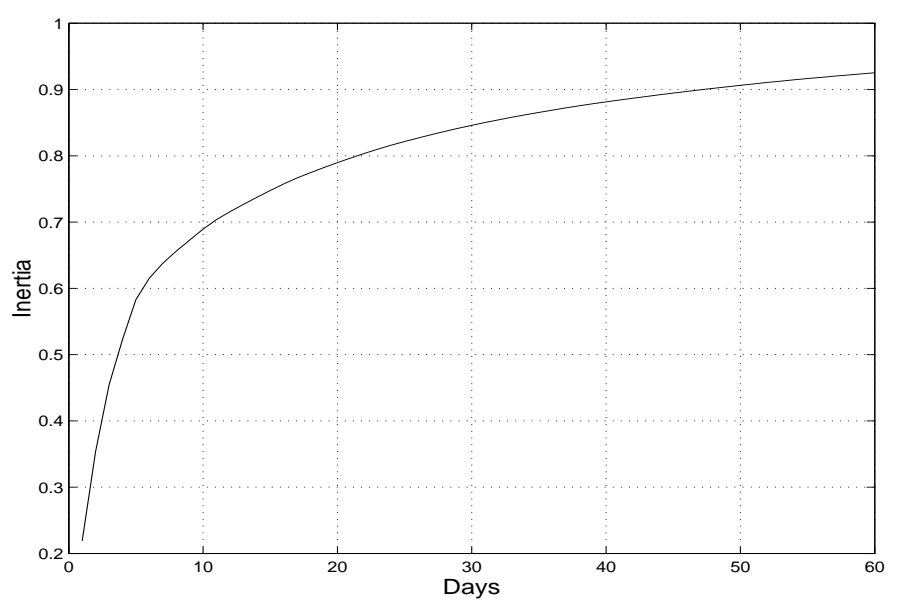

Figure 3: Percentage of inertia versus the number of retained EOFs.

inertia of the sample and this percentage is not much increased for higher value of $r_{1}$. The value of $r_{2}$ was chosen empirically to be 40 .

\subsection{Assimilation results}

In order to show the feasibility of our adaptive tuning scheme we will present the assimilation results of three experiments obtained from the ROEK filter with: (i) fixed correction basis dimension $r=35$ and fixed forgetting factor $\rho=0.8$, (ii) fixed $r=40$ and fixed $\rho=0.8$, (iii) adaptive $r=35$ or 40 and adaptive $\rho=0.5$ or 1 . The initial values of the instantaneous average $s_{0}$ and the long term average $l_{0}$ were taken as $\left\|Y_{0}^{o}-H_{k} X^{f}\left(t_{0}\right)\right\|^{2}$ to make sure that $\rho$ takes the value 0.5 during early assimilation period. The values of the constants $c, \alpha$ and $\beta$ were chosen as 1.002, 0.95 and 0.9 , respectively.

- The ROEK filter

We firstly discuss the assimilation results of the ROEK filter with a fixed number $r=35$ of retained EOFs and a fixed forgetting factor set to $\rho=0.8$. It can be seen from Figure 4 that the ROEK filter performs well both in the upper and the lower layers. Although the performances of the ROEK appears to degrade somewhat in the presence of instabilities, it still behave satisfactory during this period. One may 

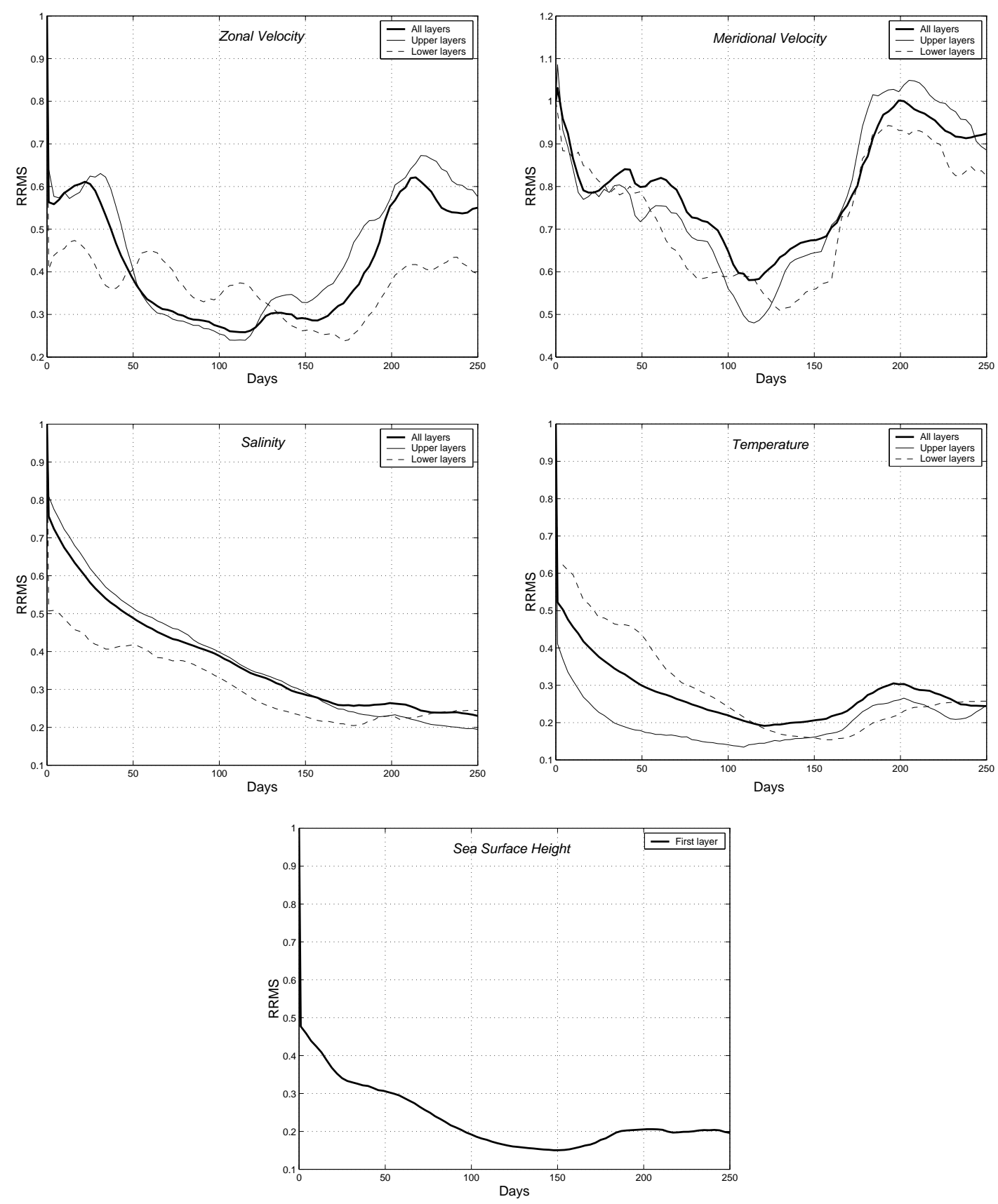

Figure 4: Evolution in time of the RRMS for the ROEK filter on the whole model domain, on the (mean of the 5) upper and the (mean of the 5) lower layers. 
think that the meridional velocity $V$ is not sufficiently well-assimilated because the assimilation error is only reduced by less than a half. But it is worthwhile to point out that, since the velocity field of the tropical Pacific ocean is particularly zonal, the meridional velocity fields are generally, and especially the referenced field in our experiment on march $1^{\text {st }} 1991$, well-approached by the average of the meridional velocity. Since this average serves as our initial analysis, the initial error is already low and therefore it would be hard to reduce it much further.

We have presented the results of our experiments for the ROEK filter in both the upper and lower layers for completeness. But we have noticed that, for the adaptive ROEK filter, the difference between their RRMS and that of the ROEK filter computed in all the layers are quite similar to that computed on each layer. Therefore, in the sequel we will only present results in all layers, to save space.

\section{- The adaptive ROEK fllter}

The assimilation results of the experiments (i), (ii) and (iii) plotted in Figure 5 and Figure 7-8, seem to confirm our theory on the number of EOFs to be retained. Indeed, in the one hand, one can see that the performance of the ROEK with $r=35$ is seriously degraded during the unstable period of the model, despite a good behavior during the stable period and, in the other hand, the performance of this filter has been improved during the model instability when more EOFs $(r=40)$ were retained but we observe the opposite phenomena in the stable period. It can also be seen that our adaptive tuning schemes based on the number of EOFs and the forgetting factor greatly enhance the performance of the ROEK filter in all the assimilation period. In particular, the feasibility of our adaptive tuning schemes of the dimension of the reduced state space and of the forgetting factor was made clear since the ROEK filter has been completely stabilized during the unstable period thanks to the increase of the value of $r$ and the decreasing value of $\rho$.

\section{Discussion}

The extended Kalman (EK) filter is one of the major tool to assimilate data into ocean models. However, its implementation in realistic ocean models is not possible because of its prohibitive cost. As proposed by Fukumori et al. [9], the use of a (linear) reduction operator, which relates the state vector of the system to a small dimension reduced-order state vector, offers us several alternatives to reduce the cost 

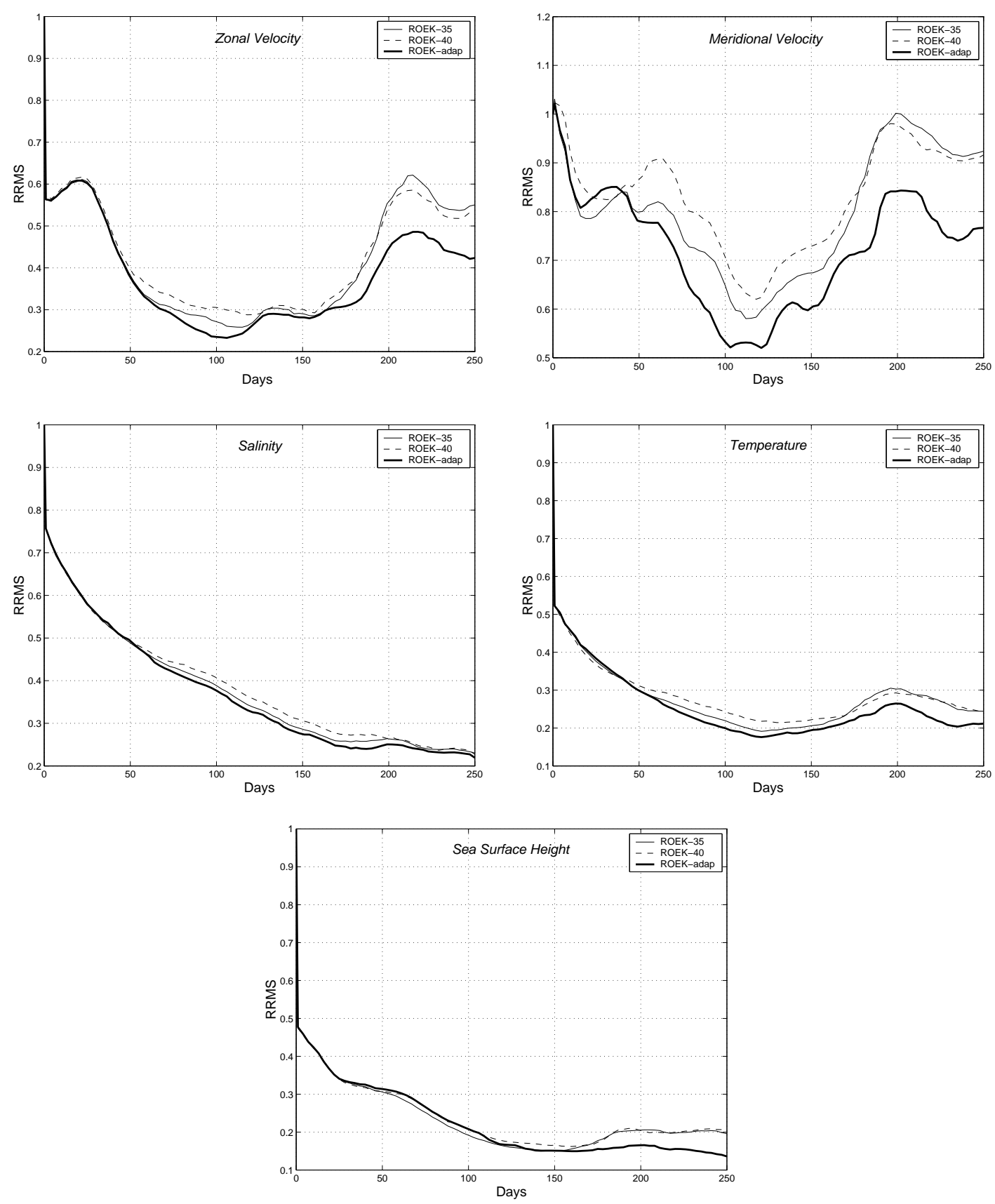

Figure 5: Evolution in time of the RRMS for the ROEK filter with and without adaptation of the forgetting factor and the dimension of the reduced state space. 


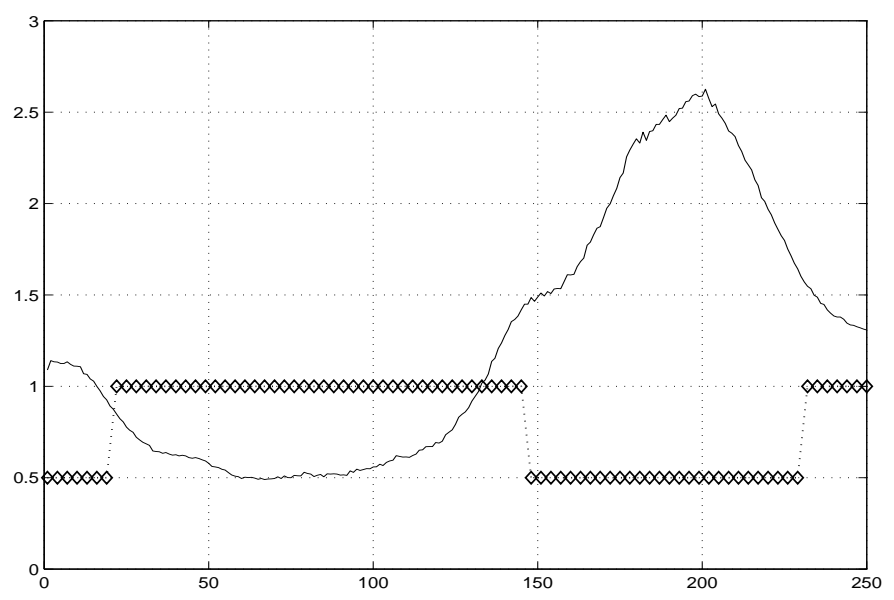

Figure 6: Evolution in time of the forgetting factor (diamond line) to show its relation with the relative variation of the state vector (the curve in Figure 2 is reproduced in solid line, rescaled by a factor of $10^{3}$ ).

of the EK filter. The resulting simplified Kalman filters have seen to be effective in assimilating data into oceanic models [3, 5, 8, 9, 18].

In the case of the ROEK filter of Cane et al. [3], where the reduced state space is obtained through and EOF analysis, it has been noticed that increasing the number of retained EOFs does not always improve the filter performance. We speculate that this comes from the fact that the EOF basis is only (at best) optimal in time average sense. We also introduce a new adaptive scheme to tune the number $r$ of retained EOFs according to the model state. Following Hoteit et al. [11, 12], our low cost scheme simply in giving $r$ one of two values according to the relative magnitudes of the instantaneous and long-term prediction errors. Note that the value of the forgetting factor has been also adapted in our adaptive ROEK filter as proposed in Hoteit et al. $[11,12]$.

The series of twin experiments which we have conducted to assess the feasibilities of our new adaptive ROEK filter and to evaluate its performance confirm our theory on the value of $r$ to be considered. Moreover, it clearly shows the feasibility of our adaptive tuning schemes in stabilizing the performance of the ROEK filter during 

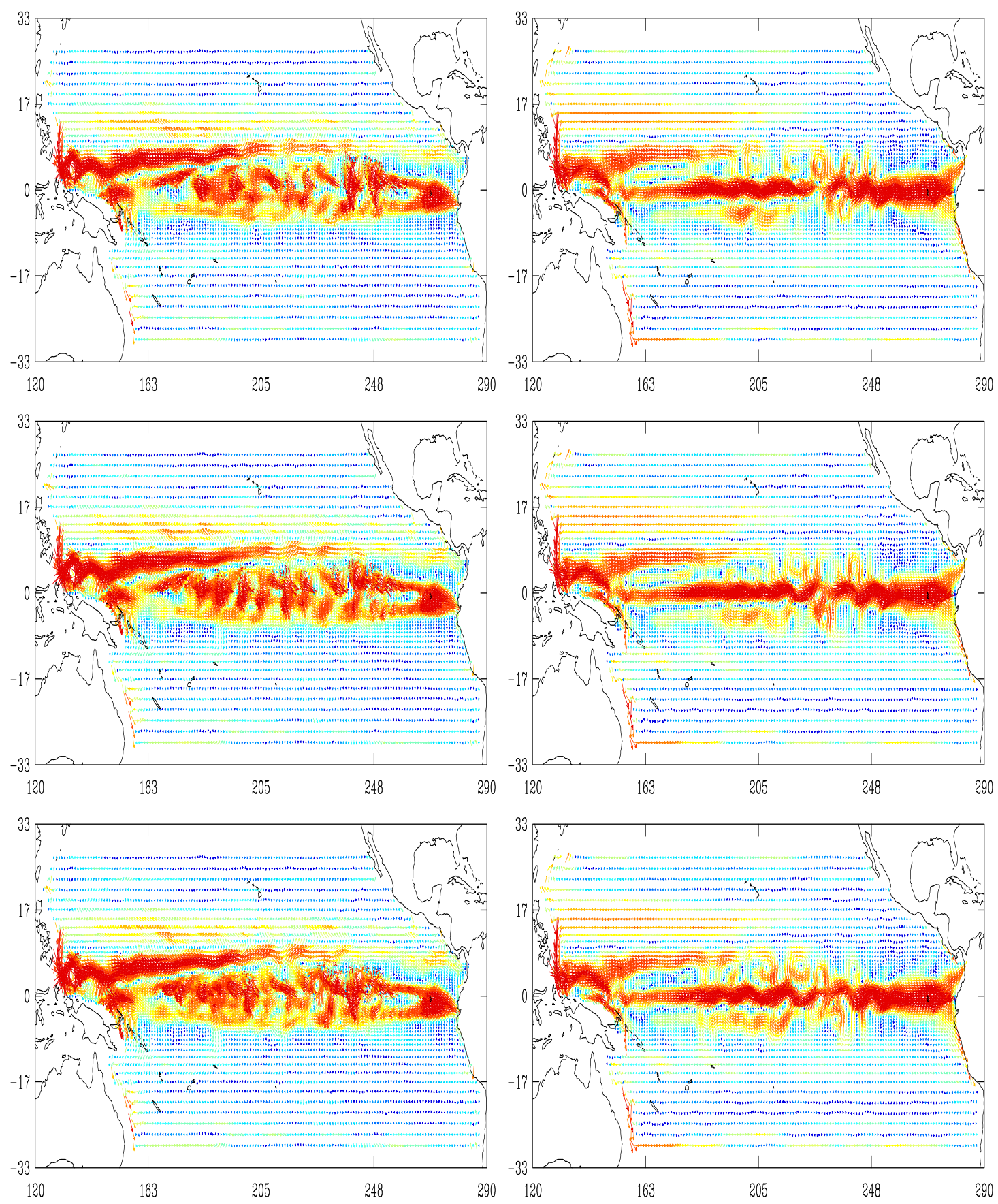

Figure 7: Maps of ocean velocity on Oct $21^{\text {st }} 90$ in the uppermost (left) and the $17^{\text {th }}$ (right) layers: from the ROEK filter (top); from the reference (middle); and from the adaptive ROEK filter (bottom).

$\operatorname{RR~} \mathrm{n}^{\circ} 4298$ 

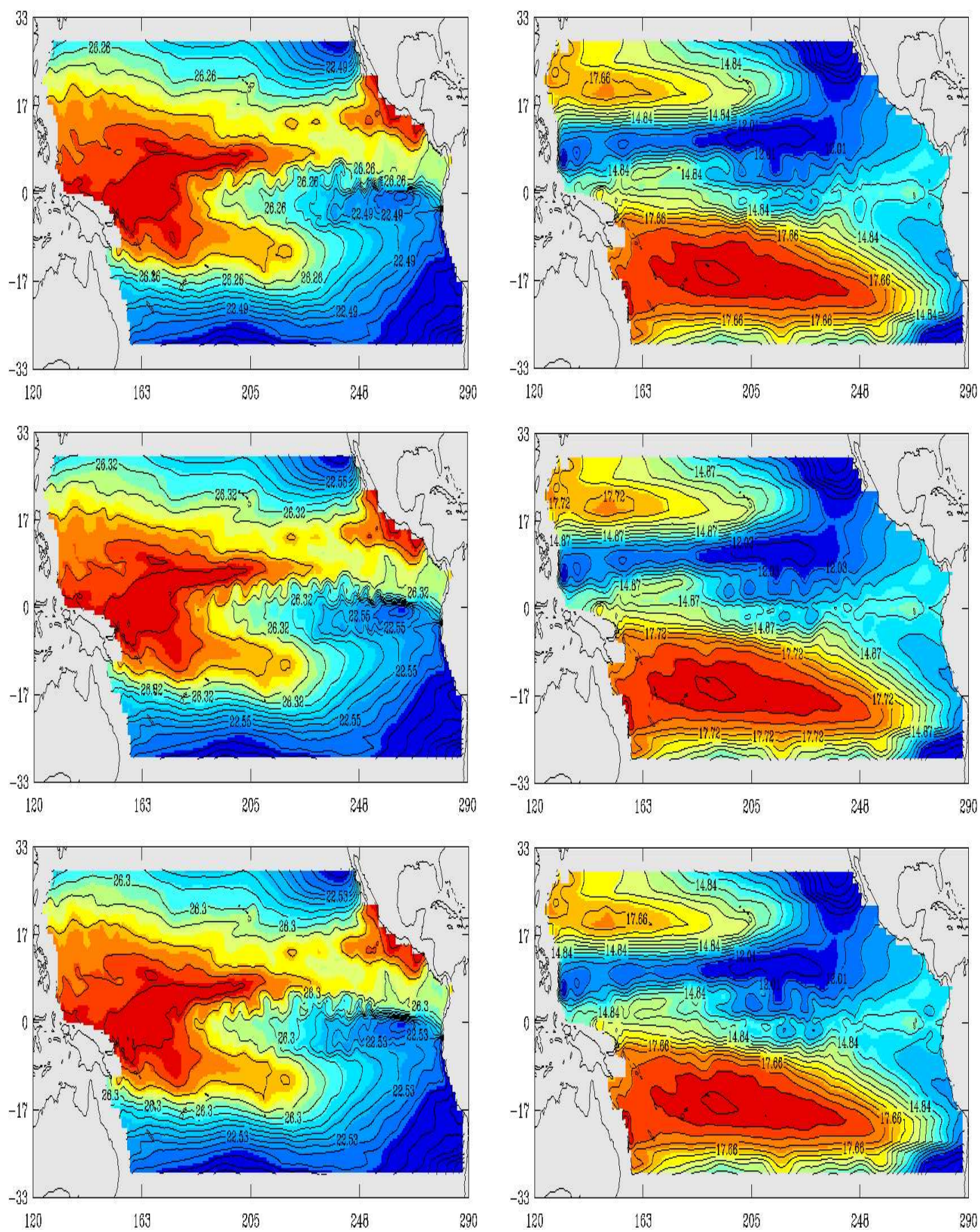

Figure 8: Maps of sea temperature on Oct $21^{\text {st }} 90$ in the uppermost (left) and the $17^{\text {th }}$ (right) layers: from the ROEK filter (top); from the reference (middle); and from the adaptive ROEK filter (bottom). 
the unstable period of the model and also in detecting such periods.

The results obtained so far are quite encouraging and suggest to examine other aspects of the EOF analysis. Indeed, since the local variability is represented by the last EOFs, it would be wiser to limit the spatial range of these EOFs since local phenomena only occur "locally". An alternative has been already proposed by Hoteit et al. [11] which have introduced the global-local EOFs (or mixed) analysis. Such analysis consists in applying a series of independent EOF analysis in the different subdomains of the model on the residue of the states in the space generated by the (classical) EOFs. A promising idea would be to use our adaptive scheme on the number of EOFs to be retained in order to tune the number of local EOFs to be considered. A work based in this idea is in progress and will be reported in the near future.

\section{References}

[1] Arakawa A. (1972): Design of the UCLA general circulation model. Numerical integration of weather and climate. Dept. of Meteorology, University of California, Rep. 7, 116 pp.

[2] Blanke B. and P. Delecluse (1993): Variability of the tropical Atlantic ocean simulated by a general circulation model with two different mixed layer physics. J. Phys. Oceanogr., 23, 1363-1388.

[3] Cane M.A., A. Kaplan, R.N. Miller, B. Tang, E.C. Hackert and A.J. Busalacchi (1996): Mapping tropical Pacific sea level: data assimilation via a reduced state Kalman filter. J. Geophys. Res., vol.101, no.C10, 599-617.

[4] Cohn S.E. and R. Tolding (1996): Approximate Kalman filters for stable and unstable dynamics. J. Meteor. Soc. Japan, 74, 63-75.

[5] Dee D.P. (1990): Simplification of the Kalman filter for meteorological data assimilation. Quart. J. Roy. Meteor. Soc., vol.117, 365-384.

[6] De Mey P. (1997): Data Assimilation at the Oceanic Mesoscale: A Review. $J$. Met. Soc. Japan, 75(1B), 415-427.

[7] Evensen G. (1992): Using the extended Kalman filter with a multilayer quasigeostrophic ocean model. J. Geophysical Research, vol.97, no.C11, 17905-17924. 
[8] Fukumori I. (1995): Assimilation of Topex sea level measurements with a reduced-gravity shallow water model of the tropical Pacific ocean. J. Geophys. Res., $\mathbf{1 0 0}(\mathrm{C} 12), 25027-25039$.

[9] Fukumori I. and P. Malanotte-Rizzoli (1995): An approximate Kalman filter for ocean data assimilation: an example with an idealized gulf stream model. $J$. Geophys. Res., 100(C4), 6777-6793.

[10] Ghil M. and P. Malanotte-Rizzoli (1991): Data assimilation in meteorology and oceanography. Adv. Geophys., 33, 141-266.

[11] Hoteit I., D.T. Pham and J. Blum (2000): A semi-evolutive partially local filter for data assimilation. Marine Pollution Bulletin (in press).

[12] Hoteit I., D.T. Pham and J. Blum (2000): A simplified reduced order Kalman filtering and application to altimetric data assimilation in tropical pacific. Technical report, RR-3937, INRIA. Submitted to J. Mar. Sys.. Available on http://www.inria.fr/RRRT/RR-3937.

[13] Ide K., A.F. Bennett, P. Courtier, M. Ghil and A.C. Lorenc (1997): Unified notation for data assimilation: operational, sequential and variational. J. Met. Soc., Japan, 75(1B), 181-189.

[14] Jazwinski A.H. (1970): Stochastic processes and filtering theory. Academic Presse, New York.

[15] Kalman R.E. (1960): A new approach to linear filtering and prediction problems. Trans. ASME Ser. D, J. Basic Eng., 82D, 35-45.

[16] Levitus S. (1982): Climatological atlas of the world ocean. Geophysical fluid dynamics laboratory, Princeton.

[17] Madec G., P. Delecluse, M. Imbard and C. Levy (1997): Ocean general circulation model reference manual. Technical report, University Pierre and Marie Curie, Paris VI.

[18] Miller R.N. and M. Cane (1989): A Kalman filter analysis of sea level height in the tropical Pacific. J. Phys. Oceanogr., 19, 773-790.

[19] Pham D.T., J. Verron and M.C. Roubaud (1997): Singular evolutive Kalman filter with EOF initialization for data assimilation in oceanography. J. Mar. Syst., vol. 16, 323-340. 
[20] Pinardi N., A. Rosati and R. Pacanowski (1995): The sea surface pressure formulation of rigid lid models: implications for altimetric data assimilation studies. J. Mar. Syst., 6, 109-119.

[21] Preisendorfer R. (1988): Principal Component Analysis in meteorology and oceanography. Elsevier Sci. Publ., 17, 425 pp. 
Unit’e de recherche INRIA Lorraine, Technopôle de Nancy-Brabois, Campus scientifique, 615 rue du Jardin Botanique, BP 101, 54600 VILLERS LÈS NANCY

Unit'e de recherche INRIA Rennes, Irisa, Campus universitaire de Beaulieu, 35042 RENNES Cedex Unit'e de recherche INRIA Rhône-Alpes, 655, avenue de l'Europe, 38330 MONTBONNOT ST MARTIN Unit'e de recherche INRIA Rocquencourt, Domaine de Voluceau, Rocquencourt, BP 105, 78153 LE CHESNAY Cedex Unit'e de recherche INRIA Sophia-Antipolis, 2004 route des Lucioles, BP 93, 06902 SOPHIA-ANTIPOLIS Cedex

Éditeur

INRIA, Domaine de Voluceau, Rocquencourt, BP 105, 78153 LE CHESNAY Cedex (France) http://www.inria.fr

ISSN 0249-6399 